\title{
Numerical Simulations and Experiments of Ignition of Solid Particles in a Laminar Burner: Effects of Slip Velocity and Particle Swelling
}

\author{
Antonio Attili, et al. [full author details at the end of the article]
}

Received: 3 December 2019 / Accepted: 29 September 2020 / Published online: 26 October 2020

(c) The Author(s) 2020

\begin{abstract}
Ignition and combustion of pulverized solid fuel is investigated in a laminar burner. The two-dimensional $\mathrm{OH}$ radical field is measured in the experiments, providing information on the first onset of ignition and a detailed characterization of the flame structure for the single particle. In addition, particle velocity and diameter are tracked in time in the experiments. Simulations are carried out with a Lagrangian point-particle approach fully coupled with an Eulerian solver for the gas-phase, which includes detailed chemistry and transport. The numerical simulation results are compared with the experimental measurements in order to investigate the ignition characteristics. The effect of the slip velocity, i.e. the initial velocity difference between the gas-phase and the particle, is investigated numerically. For increasing slip velocity, the ignition delay time decreases. For large slip velocities, the decrease in ignition delay time is found to saturate to a value which is about $40 \%$ smaller than the ignition delay time at zero slip velocity. Performing a simulation neglecting the dependency of the Nusselt number on the slip velocity, it is found that this dependency does not play a role. On the contrary, it is found that the decrease of ignition delay time induced by the slip velocity is due to modifications of the temperature field around the particle. In particular, the low-temperature fluid related to the energy sink due to particle heating is transported away from the particle position when the slip velocity is non-zero; therefore, the particle is exposed to larger temperatures. Finally, the effect of particle swell is investigated using a model for the particle swelling based on the CPD framework. With this model, we observed negligible differences in ignition delay time compared to the case in which swelling is not included. This is related to the negligible swelling predicted by this model before ignition. However, this is inconsistent with the experimental measurements of particle diameter, showing a significant increase of diameter even before ignition. In further simulations, the measured swelling was directly prescribed, using an analytical fit at the given conditions. With this approach, it is found that the inclusion of swelling reduces the ignition delay time by about $20 \%$ for small particles while it is negligible for large particles.
\end{abstract}

Keywords Coal combustion $\cdot$ CPD $\cdot$ Numerical simulartion $\cdot$ Experiment 


\section{Introduction}

Oxy-fuel combustion combined with solid fuels is a promising approach for carbon capture and storage (CCS) to sequestrate and reduce carbon dioxide $\left(\mathrm{CO}_{2}\right)$ emissions. In an oxyfuel burner, pulverized solid particles burn in a mixture of oxygen and recycled flue gas (mainly $\mathrm{CO}_{2}$ ) instead of air used in conventional burners. Several experimental (Molina and Shaddix 2007; Bejarano and Levendis 2008; Shaddix and Molina 2009; Liu et al. 2011; Khatami et al. 2012; Riaza et al. 2014) and numerical (Maffei et al. 2013; Tufano et al. 2016; Jimenez and Gonzalo-Tirado 2017) studies indicate, for example, that replacing $\mathrm{N}_{2}$ by $\mathrm{CO}_{2}$ increases the ignition delay time and duration of devolatilization.

Recently, numerical simulations for single (Farazi et al. 2019a) and multiple particles (Farazi et al. 2019b) have been performed employing the chemical percolation devolatilization (CPD) model (Grant et al. 1989) in a fully coupled Eulerian-Lagrangian pointparticle numerical framework to investigate a number of aspects including the sensitivity of ignition delay time to temperature, gas composition, and particle number density. In addition to the aforementioned parameters, the particle size, the slip velocity, i.e., the difference between the particle and the surrounding gas velocity, the particle swelling and other variables play a role in controlling ignition and combustion.

In the present study, the ignition of solid particles is analyzed comparing detailed experimental measurements Köser et al. (2019) of the spatial distribution of $\mathrm{OH}$ around the particles with highly resolved numerical simulation employing the Chemical Percolation Devolatilization (CPD), finite rate chemistry, and a Lagrangian point-particle approach for the dispersed phase. In particular, experimental measurements provide time resolved data for the evolution of the particle velocity, size, and finally ignition delay time for a large number of conditions and initial particle sizes. Combining parametric variations of these parameters in the simulations and experimental results, it is possible to assess the effects of the slip velocity, swelling, and particle size and to understand the mechanisms responsible of the observed behavior. It is worth noting that using a point-particle approximation, while not providing the full details of a particle resolved method, allows to analyze and validate the different phenomena and models in the same framework that is typically employed in more complicate settings, such as turbulent and multiple particle flows, which cannot be simulated by the particle resolved method.

The paper is organized as follows. In Sect. 2, the experimental configuration and methods are presented first, followed by description of the numerical approach. In Sect. 3, a comparison between the measured and computed ignition delay times for different particle sizes are presented and the effect of slip velocity between the particle and gas phase and the impact of particle swelling are assessed leveraging simulation results for a number different cases.

\section{Methods}

\subsection{Experimetal Methodology}

The experiments were conducted in an enclosed laminar flow reactor, as schematically shown in Fig. 1a. The reactor is the same as that described in details by Köser et al. (2019). It is composed of a particle seeder with a $0.8-\mathrm{mm}$ injection tube, a ceramic honeycomb 


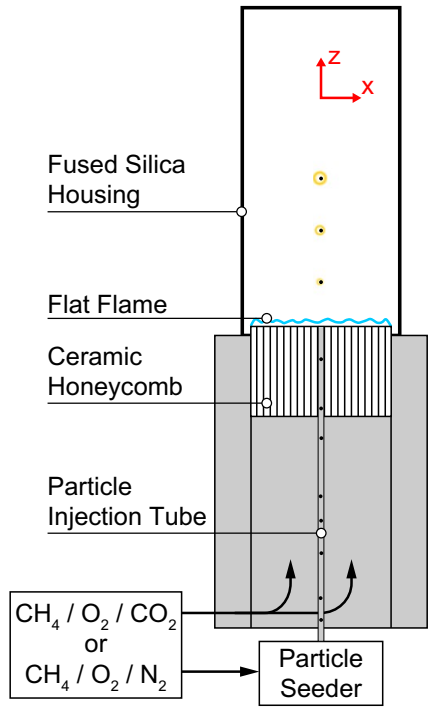

(a)

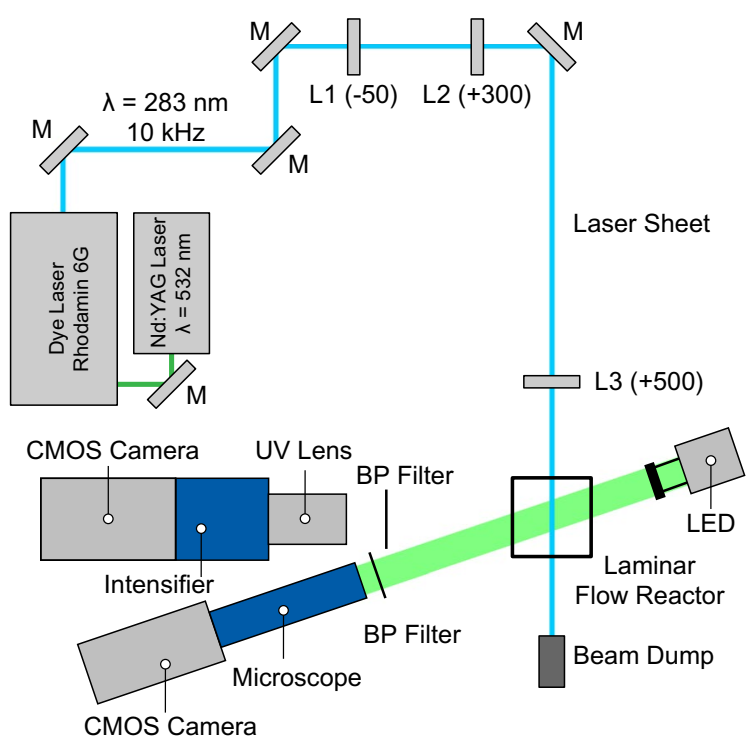

(b)

Fig. 1 a Sketch of experimental setup. b Sketch of laminar flow reactor

$\left(\sim 80 \times 80 \mathrm{~mm}^{2}\right)$ and fused silica chimney enclosure. A laminar premixed flame was stabilized $\sim 1.5 \mathrm{~mm}$ above the honeycomb top surface. The reactor was operated at different airconditions (inlet mixture of $\mathrm{CH}_{4} / \mathrm{O}_{2} / \mathrm{N}_{2}$ ) with oxygen concentration of $10 \mathrm{vol} \%, 20 \mathrm{vol} \%$, $30 \mathrm{vol} \%$, and $40 \mathrm{vol} \%$ in the exhaust gas and oxy-conditions (inlet mixture of $\mathrm{CH}_{4} / \mathrm{O}_{2} /$ $\mathrm{CO}_{2}$ ) with oxygen concentration of $20 \mathrm{vol} \%, 30 \mathrm{vol} \%$, and $40 \mathrm{vol} \%$ in the exhaust gas. In the present work, the analysis focuses on the Air-condition with an oxygen concentration of $20 \mathrm{vol} \%$. The measured gas temperature was about $1800 \mathrm{~K}$ directly downstream of the premixed flame and showed minor variation in different operating conditions, as reported by Köser et al. (2019). Particles were injected through the central tube supported by the carrier gas with the same mixture as the flat flame inlet. The heat-up point was well defined by the particle crossing the enclosed premixed flame front and the steep rise of the gas temperature. The quadratic quartz glass enclosure enabled free optical access from all sides of the reactor.

The coal investigated in this study is high-volatile bituminous Colombian coal. The proximate analysis of the coal composition was $3.5 \%_{m}$ moisture(an), $36.9 \%_{m}$ volatiles(wf), $54.4 \%_{m}$ Cfix(wf) and $8.7 \%_{m}$ ash(wf) (Vorobiev et al. 2016). Various samples with size varying from 90 to $125 \mu \mathrm{m}$ were used to investigate the ignition and volatile combustion of single coal particles.

The laser-induced fluorescence of $\mathrm{OH}$ radicals (OH-LIF) was employed to investigate the volatile combustion process of single coal particles. The experimental setup is illustrated in Fig. 1b. A frequency-doubled dye laser (Sirah Credo, Rohdamin 6G) was pumped by a diode-pumped solid-state laser (EdgeWave IS8II-E) at $532 \mathrm{~nm}$. The dye laser was tuned at $283.01 \mathrm{~nm}$ to excite the $Q_{1}(6)$ line of the A-X (1-0) transition of $\mathrm{OH}$ radicals. The laser was operated at $10 \mathrm{kHz}$ repetition rate and produced an average pulse energy of $\sim 0.33 \mathrm{~mJ}$. The laser was expanded to a $\sim 20 \mathrm{~mm}$ high sheet and focused at the burner center line with a thickness of $\sim 100 \mu \mathrm{m}$. The $\mathrm{OH}$ fluorescence signals were collected by 
a CMOS camera (LaVision HSS6) coupled with a two-stage intensifier (LaVision HSIRO). The camera was equipped with a UV-achromatic lens (Halle, $f=150 \mathrm{~mm}, \mathrm{f} / 2.5$ ). A band-pass filter $(320 \pm 20 \mathrm{~nm})$ and an intensifier gate of $100 \mathrm{~ns}$ were applied to suppress broadband chemiluminescence and thermal radiation. With the projected pixel resolution of $27.55 \mu \mathrm{m} /$ pixel, the optical resolution was estimated to be about $\sim 100 \mu \mathrm{m}$ by imaging a Siemens star. The ignition delay time was detected by evaluating the Pearson's correlation coefficient $R$ of temporal OH-LIF signals. A small region of interest (ROI) of $200 \times 200$ pixels centered on the particle centroid was extracted for instantaneous $\mathrm{OH}$ images. The $\mathrm{R}$ calculation restricted in the defined ROI was performed between each instantaneous image and the last image of a LIF sequence showing evident spacial volatile flame structure. The ignition delay time was defined as the first time when $\mathrm{R}$ exceeds 0.8 indicating strong correlations. The method was validated against the algorithm proposed in Köser et al. (2019) and showed consistent results.

A high-speed diffusive backlight-imaging (DBI) system was employed to measure the in-situ particle diameter and velocity. A high-power LED (LPS, ILA) was operated at $10 \mathrm{kHz}$ with a pulse duration of $1 \mu \mathrm{s}$ and temporally synchronized with the OH-LIF imaging system. The DBI system was inclined by $\sim 17^{\circ}$ to the OH-LIF camera, which is shown in Fig. 1b. To detect the backlight image of particles, a CMOS camera (Phantom v711) was equipped with a long-distance microscope (Infinity, SK2). A band-pass filter $(525 \pm 20 \mathrm{~nm})$ suppressed the broadband radiation of sooting and burning particles. The projected pixel size was $9.2 \mu \mathrm{m}$. The DBI images were first binarized by defining the particle boundary using canny detection method and then the centroid and area of particle were calculated. The equivalent diameter was calculated based on a circle with the same area as the particle. The particle velocity was derived from centroid position using a fivepoint stencil. A detailed description of the DBI approach is provided by Li et al. (2020). In particular, the procedure employed to compute the particle diameter is explained and the accuracy of this procedure is assessed carefully.

\subsection{Models and Configurations for the Numerical Simulations}

In the present work, coal combustion is modeled in an Euler-Lagrange framework, employing a point-particle approximation for the dispersed solid phase. In the previous study of Farazi et al. (2019a), the accuracy of the applied models and methods has been validated against experimentally measured ignition delay time data by Liu et al. (2011). The governing equations in the gas phase, governing a gas mixture obeying the ideal gas law in the low-Mach number approximation, are described in detail by Attili et al. (2014). Chemical reactions in the gas phase are modeled with finite rate chemistry adopting the GRI 3.0 mechanism (Smith et al. 2017). The accuracy of the GRI mechanism for the fuel composition and concentration usually encountered in the present context has been investigated before and summarized in the supplementary material of Farazi et al. (2019a). In particular, a larger mechanism validated for heavier fuels (Bisetti et al. 2012) has been compared with GRI and negligible differences in the ignition delay time have been observed. Gas-phase radiation is included using a optically-thin approximation. The Euler equations in the gas phase are solved using a semi-implicit finite difference code, with 2nd order accuracy in space and time (Desjardins et al. 2008). The solution is advanced in time with the Crank-Nicolson method along with an iterative predictor-corrector scheme (Farazi et al. 2017). 
Coal is modeled with point particles within the Lagrangian framework. Since the basic equations and models employed in this work are available in previous publications, only a summary is included here, while the models that are relevant for the discussion in the following sections, such as the swelling model, are described in detail. Particle equations are available in the classical paper of Miller and Bellan (1999). The trajectory, velocity, mass, and temperature of each particle are computed from the equations reported by Farazi et al. (2019b). To compute the devolatilization rate for each particle, the Chemical Percolation Devolatilization (CPD) model (Grant et al. 1989) is applied. Applying the CPD model, the release rate and composition of the light gases are computed as a function of time according to the study by Jupudi et al. (2009). In the present work, the light gases consist of $\mathrm{CH}_{4}, \mathrm{CO}, \mathrm{H}_{2} \mathrm{O}, \mathrm{CO}_{2}$, and "other gases". We assumed the "other gases" calculated by CPD to be $\mathrm{C}_{2} \mathrm{H}_{2}$, as done also by Jimenez and Gonzalo-Tirado (2017), Farazi et al. (2019a), and Goshayeshi and Sutherland (2014). The rate of the tar released is also computed by the CPD model and it is assumed that tar is only $\mathrm{C}_{2} \mathrm{H}_{2}$. This assumption has been used also by Goshayeshi and Sutherland (2014) and Farazi et al. (2019a). To assess the effect of this assumption on ignition delay, we also employed $\mathrm{C}_{6} \mathrm{H}_{6}$ as tar species instead of $\mathrm{C}_{2} \mathrm{H}_{2}$ and we observed only a marginal difference in ignition delay time.

Since measurements of the evolution of the particle diameters are available from the experiments, a model for the particle swelling has also been tested. Similarly to the devolatilization rate, the model for swelling is based on the CPD framework (Shurtz et al. 2011, 2012). The particle diameter is described as a function of the internal structure of the particle based on the CPD model. A swelling factor is assumed to be related the time derivative of the particle temperature, i.e., the particle heating rate, obtained from the particle energy balance (Farazi et al. 2019b). In the model of Shurtz et al. (2011, 2012), two regimes are considered for different particle heating rates $\dot{T}$, below and above $10^{4} \mathrm{~K} / \mathrm{s}$, respectively. For both regimes, the swelling ratio can be computed as function of the heating rate and the time evolution of the internal variables of the CPD model. In the CPD model, the internal variable $l$ represents the labile bridge fraction in the polymer network. During devolatilization, the labile bridges are converted to reactive intermediates that can either cleave into two side chains or react to form stabilized char bridges and light gases. As the labile bridge fraction decreases from its initial value to zero, the polymer network of the coal is converted to a highly cross-linked char structure, which would tend to limit swelling. Therefore, at the end, increase in the particle diameter is usually delayed until starting of the devolatilization process (Shurtz et al. 2011, 2012).

It is worth noting that the particles are assumed to be spherical in the present work. While this might be a good approximation, it is certainly true that the effects of the actual shape on ignition and combustion deserve additional investigation. However, this is outside of the scope of the present work, but it will be considered in future publication since the imaging technique employed in the experiment can be used to extract the particle shape.

In the swelling model, the diameter $D_{p}$ of the particle is assumed to be governed by the following equations:

$$
D_{p}=D_{p_{0}}\left(1+\operatorname{swell}\left[1-l / l_{0}\right]\right)
$$

with $l_{0}$ and $l$ being the initial and current fraction of labile bridges and $D_{p_{0}}$ the initial particle diameter. swell is a funtion of the heating rate $\dot{T}$ :

$$
\text { swell }=s_{\text {var }}\left(\frac{\dot{T}_{\text {Base }}}{\dot{T}}\right)^{C_{H R}}+s_{\text {min }}-1
$$


for high heating rates and as

$$
\text { swell }=m \log (\dot{T})+b-1
$$

for low heating rates. The coefficients $\dot{T}_{B a s e}, s_{v a r}, C_{H R}, s_{\text {min }}, b, m$ in the swelling equations above can be evaluated from the input parameters of the CPD model following Shurtz et al. (2011, (2012).

The gas phase is coupled to the particles through the source terms appearing in the conservation equations. For a general case of a simulation with a certain number of particles (Farazi et al. 2019b), a distribution coefficient $\phi_{k}$ is employed for the source terms of the $k$ th particle. $\phi_{k}$ is computed from a Gaussian function with a characteristic width $L_{d}$, centered at the position of the $k$ th particle. This approach has been successfully employed before by Apte et al. (2008) and Farazi et al. (2019a). Here, the distribution length $L_{\mathrm{d}}$ is set to $2 D_{p}$.

The quantities required from the gas phase are averaged within a cube with side length $L_{\mathrm{f}}$, centered at the particle location. Sensitivity studies showed that the filter length does not have a significant effect on the results.

The domain is discretized using a uniform grid consisting of cubic cells with size $d x$ equal to the particle diameter $D_{p}$. A convergence study showed that for $d x<D_{p}$ the computed ignition delay time does not change. Therefore, we performed all simulations with $d x=D_{p}$. A careful assessment of this approach is discussed by Farazi et al. (2019a), where it is shown that the methods used here are appropriate for accurate simulations of particle ignition.

For the numerical simulations, an inlet outlet configuration is considered. A gas-stream in injected on the left side of the domain with thermochemical conditions obtained from an unstretched laminar premixed flame. In particular, the fully burned gas composition and temperature obtained in the premixed flame are employed. Initial velocity, temperature, size, and composition of the particle are prescribed and evolve due to the interaction with the surrounding gas. The approximation of neglecting the region where the particle crosses the premixed flame is expected to have a negligible influence because the thickness of the premixed flame is small and then the corresponding residence time of the particle inside the flame region is short compared to the overall ignition delay time.

\section{Results}

\subsection{Overall Analysis of the Ignition Process and Volatile Combustion}

Figure 2 exemplarily shows six temporally sequential $\mathrm{OH}$ images in the early stage of gas phase combustion measured in the experiment. The particle for this case is in the size range $90-125 \mu \mathrm{m}$ and the atmosphere with $20 \%$ in volume of oxygen is used. The $\mathrm{OH}$ signal, which depends on the local reactivity and temperature, is treated as an indicator of the reaction zone. The detected $\mathrm{OH}$ signals were corrected regarding the laser sheet inhomogeneity and pulse energy fluctuations. A long dark streak on the right-hand side is observed indicating that the particle is located approximately centrally in the laser sheet. In these images, the region shown is always centered at the particle location. The ignition time $t_{0}$ is defined as the instant when the first increase of signal intensity against background level is observed. The first apparent $\mathrm{OH}$ signals are observed above the particle implying that the mixture of outgassing volatile and oxidizer has higher 

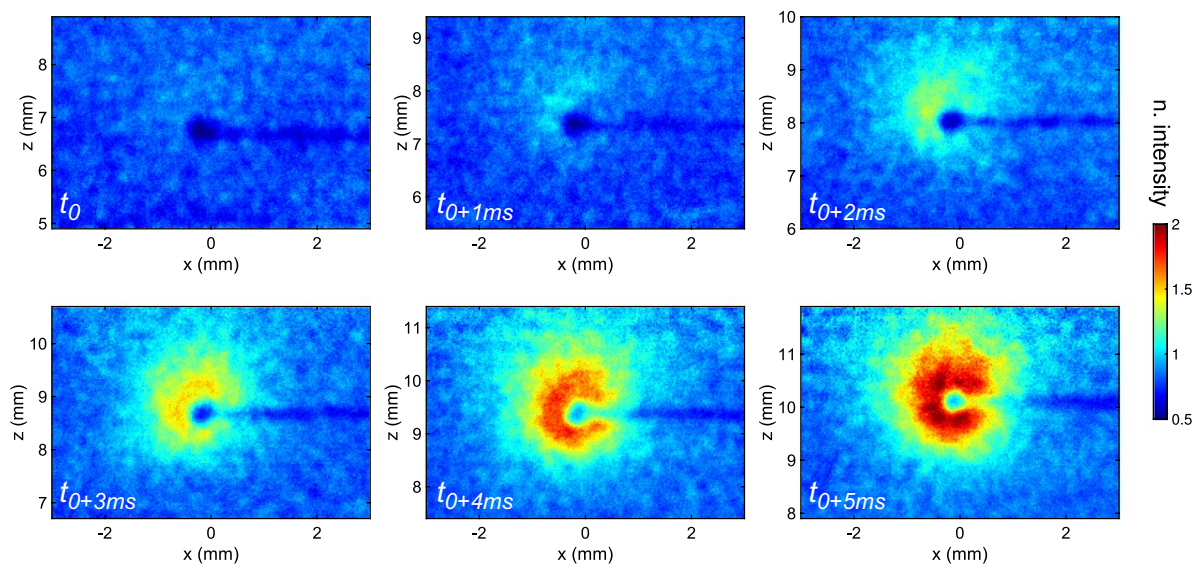

Fig. 2 The temporally sequential OH-LIF images of a single particle volatile flame. The initial particle diameter in this case is in the range $90-125 \mu \mathrm{m}$. $t_{0}$ indicates the instant of ignition, which is $7 \mathrm{~ms}$ after paricle injection in this case. The flow goes from bottom to top. The dark streak is a shadow caused by the particle

chemical reactivity in this region, caused by proper mixing and high temperature. The ignition location is affected by non-isotropic pyrolysis processes during particle heatup and the particle motion (slip and rotation) (Köser et al. 2019). In the subsequent frames, the particle is enclosed by a spherical flame, whereas some inhomogeneity of $\mathrm{OH}$ spatial distribution still exists. This can be explained by the continued devolatilization process after ignition, which can also be accelerated by thermal irradiation from hot regions in the neighbouring gas phase, leading to an inhomogeneous mixture field supporting a non-spherical diffusion flame. In addition, a consistent increase of intensity and reaction zone volume is observed as the volatile combustion becomes more intense. The full development of an enveloping volatile flame occurs within a few milliseconds as discussed in previous work (Köser et al. 2017). Combined with other laser measurements such as luminosity imaging, also the end of the volatile flame can be captured. The entire duration of gas phase flame can be investigated for different particle size and oxygen concentration. Moreover, in situ particle motion measurements using diffusion-backlight illumination also contribute to the understanding the evolution of volatile flame.

The numerical approach described in the previous section has been applied for the condition of the experiment reported above. An unstretched laminar flame simulation provides the following fully burned condition to be used as inflow condition in the particle ignition simulation resulting in a gas temperature of $1815.47 \mathrm{~K}$, and a gas-phase composition of: $Y_{\mathrm{O}_{2}}=0.2242, Y_{\mathrm{H}_{2} \mathrm{O}}=0.0851, Y_{\mathrm{CO}_{2}}=0.1047, Y_{\mathrm{N}_{2}}=0.586$, plus minor amounts of additional species. These thermo-chemical conditions will be used for all simulations presented in the following analysis, while the diameter of the injected particles will be varied parametrically. In addition, different inlet particle velocities will be considered and the effect of swelling will be assessed. After the particle is injected at the domain inlet, it undergoes a phase of temperature increase due to the energy transfer from the gas-phase, which is injected at high temperature. Due to the increase of the particle temperature, volatile gases start to be released from the particles and mix with the high-temperature oxidizing environment surrounding the particles. Due to the high temperature ignition occurs once the local 


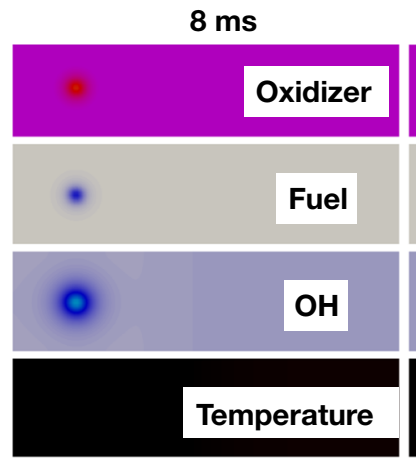

$11.5 \mathrm{~ms}$

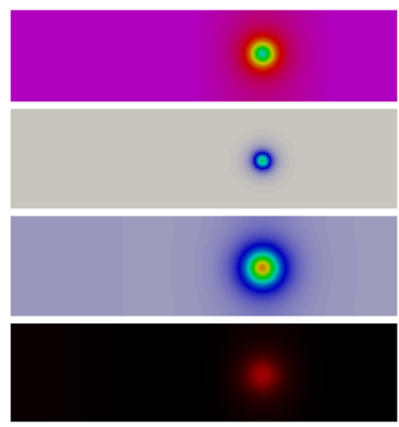

$9 \mathrm{~ms}$

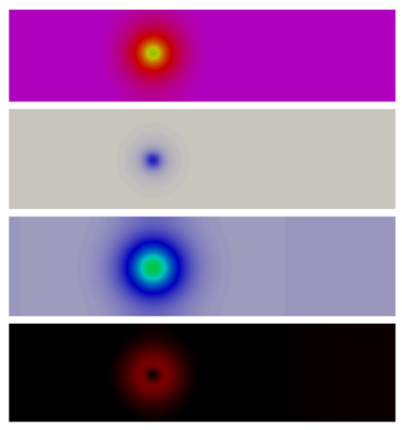

$12.5 \mathrm{~ms}$

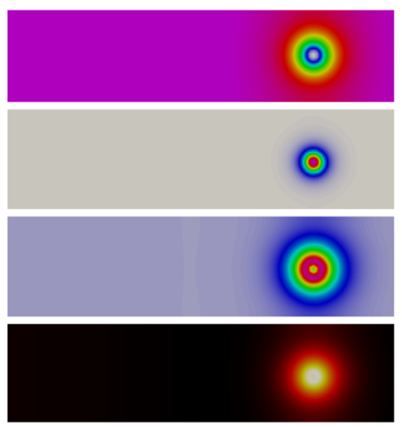

$10 \mathrm{~ms}$

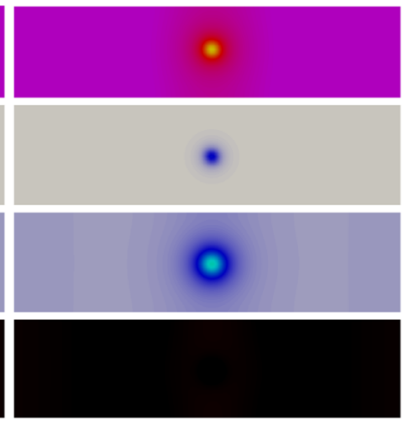

Oxidizer

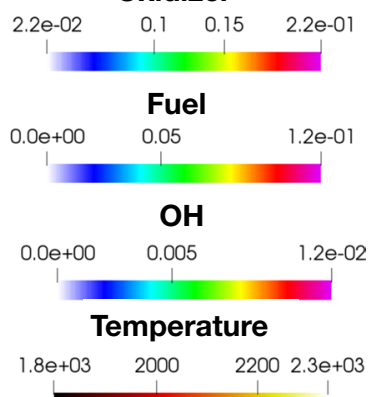

Fig. 3 Simulation results for the evolution of oxygen, volatile gas (fuel) released from the particle, $\mathrm{OH}$ radi$\mathrm{cal}$, and temperature during the ignition of a coal particles with initial diameter of $107 \mu \mathrm{m}$. The results are shown for different time instants, measured from the time at which the particle is injected in the domain. The same region of the domain, which is a small portion compared to the overall domain size, is shown at every time instant. In this case, the ignition delay time is observed after $6.5 \mathrm{~ms}$

concentration of fuel released from the particle is high enough. Figure 3 shows selected gas-phase fields around the particle at different time instants during particle ignition.

Figure 4 shows the typical time evolution of the peak $\mathrm{OH}$ mass fraction and peak heat release rate over the entire domain of the simulation, starting from the time at which the particle is injected. The time of ignition, identified based on the evolution of both the $\mathrm{OH}$ mass fraction and the heat release rate, is also shown in the figure. In particular, the difference between the ignition delay times computed from $\mathrm{OH}$ and heat release rate provides an uncertainty range for the results shown in the following. After ignition, the rate of volatile release from the particle increases and combustion is sustained for approximately $15 \mathrm{~ms}$ at the conditions considered in this case. After the volatiles have been completely released, combustion ends since char burnout is not included in the present simulation. Including burnout would not change the conclusion presented in the following, since the analysis is restricted to the ignition process and to the very early phase of volatile combustion, when char burnout is insignificant.

An overall comparison of the measured ignition delay time and that computed in the simulation is presented in Fig. 5 for particles characterized by different initial diameter. In this comparison, the initial particle diameter is used to plot the experimental results. For these simulations, swelling is not considered, so the diameter of the 


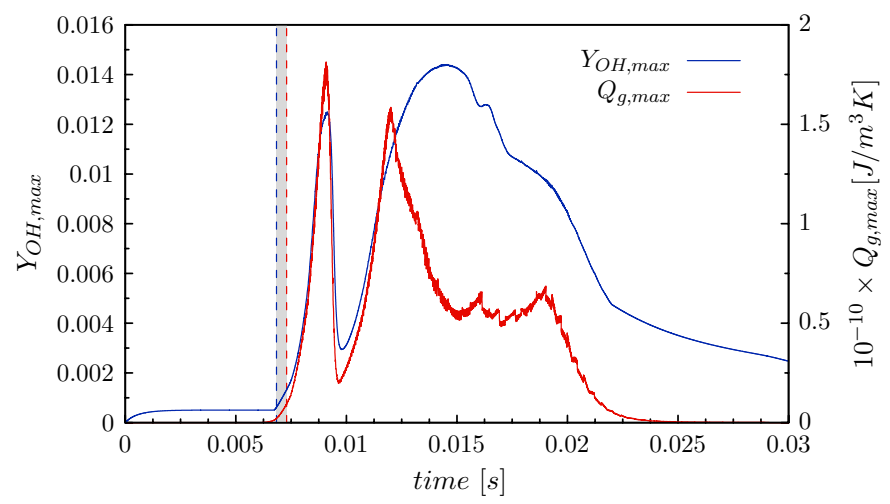

Fig. 4 Simulation results for the time evolution of peak $\mathrm{OH}$ mass fraction and peak heat release rate over the entire domain. Time is measured from the instant at which the particle is injected in the domain. The vertical dashed lines indicate the time of ignition based on $\mathrm{OH}$ and heat release, while the shaded area marks the uncertainty based on the use of the two different criteria. The initial particle diameter is $107 \mu \mathrm{m}$

Fig. 5 Ignition delay times measured in the experiment (black diamonds) and computed in the simulations (red circles) versus the initial particle diameter. Each black diamond correspond to the measurement of a single particle ignition. The error bars in the numerical results are defined by the difference in ignition delay time based on different variables and marked by the shaded area in Fig. 4. In the numerical simulations, the initial velocity of the particle is equal to the gas velocity in these simulations

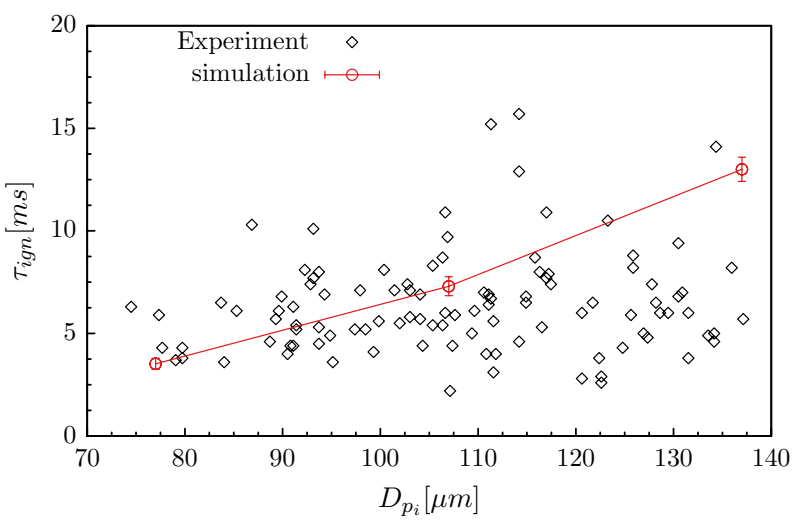

particles is constant. In addition, the initial particle velocity is assumed to be equal to the gas phase velocity at the inlet. As shown already in previous studies using the same simulation framework (Farazi et al. 2019a), the ignition delay time $\tau_{i g n}$ increases for larger particle diameter. An increase of the ignition delay time with the diameter is observed in both experiments and simulations. The observed increase of $\tau_{i g n}$ is due to the increased time needed to heat up larger particles to a temperature at which significant release of volatiles occurs. A significant scatter is observed in the experimental measurements. The scatter is related to the uncertainty in the initial particles diameter, which in the experiment is directly measured with a two-dimensional optical technique. However, due to the fact that the particles are generally not spherical, the measured size depends on the particle orientation with respect to the plane of measurement. 


\subsection{Effect of the Slip Velocity}

In addition to the ignition delay time, particle size, and OH-LIF, experimental data for the time evolution of the particle velocity are also available. In combination with the numerical simulations, it is then possible to investigate the effect of the difference between the particle and gas velocity on the computed ignition delay time.

First, the simulations presented in Fig. 5 were repeated removing the assumption that the initial particle velocity matches that of the gas. In particular, the measured values of the particle velocity for the given particle diameter were used. For the given diameter $D_{p}$ of the simulated particle, an average value of the particle velocity, obtained among all measured particles with a diameter in a small range around $D_{P}$, was imposed as initial condition for the simulations. The effect on the ignition delay time of prescribing the experimentally measured initial particle velocity instead of assuming a zero slip velocity is shown in Fig. 6. Considering slip velocity, it is found that the ignition delay time is reduced. The simulated time evolution of the particle velocity for different initial particle diameters is shown in Fig. 7. A case with an initial particle velocity equal to that of the gas (initial zero slip velocity) is also shown. Finally, the results for an initial particle diameter of 107 $\mu \mathrm{m}$ are also directly compared with the experimental measurements of the velocity. The experimental result is obtained averaging the measured velocity among a number of particle trajectories with the initial particle diameter in the range 90-125 $\mu \mathrm{m}$. For all cases with non-zero initial slip velocity, the particle velocity tends to approach the gas velocity, which is equal to $1.5 \mathrm{~m} / \mathrm{s}$ for all cases in this figure. The rate at which the particle velocity approaches that of the gas depends on the particle diameter, but in all cases the slip velocity remains significant up to the time of ignition.

Since it is found that an initial slip velocity has an effect on the ignition time, which has been observed also in an other study (Farazi et al. 2019b) where the ignition of a stream of multiple particles has been analyzed, it is of interest to investigate this dependency in a

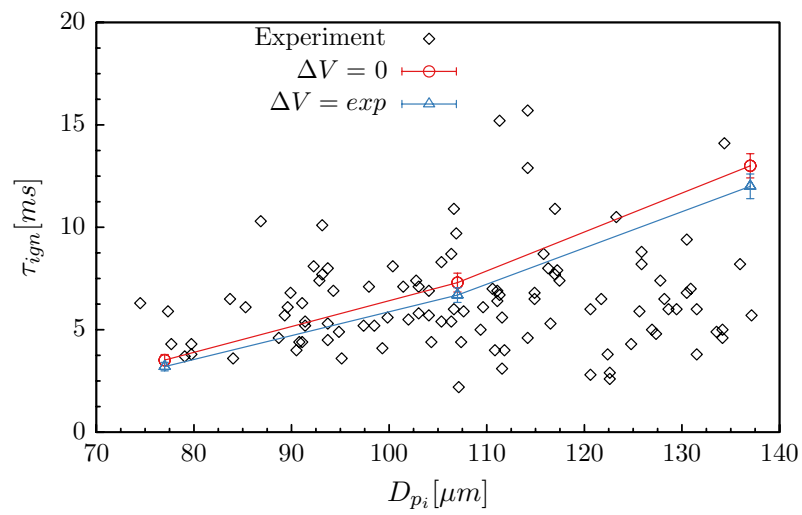

Fig. 6 Ignition delay times measured in the experiment (black diamonds) and computed in the simulations with an initial particle velocity identical to the gas velocity $\left(V_{g}=1.5 \mathrm{~m} / \mathrm{s}\right)$, i.e., zero slip velocity (red circles) and with the initial velocity measured in the experiment, i.e. non-zero slip velocity (blue triangles) versus the initial particle diameter. For the cases with non-zero slip velocity, the values for the initial particle velocity in the simulations are $0.4 ; 0.8 ; 1.0 \mathrm{~m} / \mathrm{s}$, for initial particle diameters $77 ; 107 ; 137 \mu \mathrm{m}$ respectively, while the inlet gas velocity is always $1.5 \mathrm{~m} / \mathrm{s}$. Each black diamond correspond to the measurement of a single particle ignition. The error bars in the numerical results are defined by the difference in ignition delay time based on different variables and marked by the shaded area in Fig. 4 


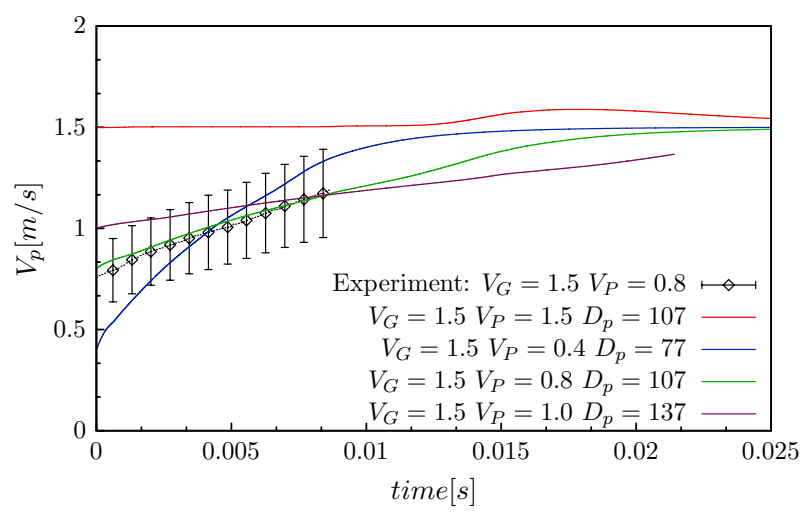

Fig. 7 Time evolution of the particle velocity for different simulation cases: initial zero slip velocity for a particle with initial diameter of $107 \mu \mathrm{m}$ (red line) and with non-zero slip velocity as explained in the caption of Fig. 6. An example of the time evolution of the particle velocity in the experiment is also shown (black diamonds). For the experiments, the symbols indicate an average among several particles with initial diameter in the range $90-125 \mu \mathrm{m}$, while the error bar is the corresponding variance

systematic way and analyze the mechanisms responsible for the dependency of the ignition delay time to the slip velocity.

A series of simulations has been conducted for different values of the initial slip velocity fixing all other parameters and conditions, including the initial particle diameter. Two different ways of imposing the slip velocity are considered. The first is that already used above; in this case the particle is injected into the domain with a velocity that differs from that of the gas. The particle velocity is free to adjust towards the value of the gas velocity. This configuration will be referred as "moving particle". In the second case, the slip velocity is kept constant. This is done by keeping the particle location fixed in a domain with a gas velocity corresponding to the desired constant slip velocity. This configuration will be referred as "fixed particle".

A summary of the results for the ignition delay time for this set of cases is shown in Fig. 8. The slip velocity $\Delta v$ is defined as the difference between the gas velocity $V_{g}$ and the particle velocity $V_{p}$. For the "moving particle" cases, the gas velocity at the inlet is always

Fig. 8 Dependency of the ignition delay time on the slip velocity obtained with fixed particles with constant slip velocity (red circles) and moving particle with different diameters (black diamonds and blue triangles). The error bars are defined by the difference in ignition delay time based on different variable and marked by the shaded area in Fig. 4

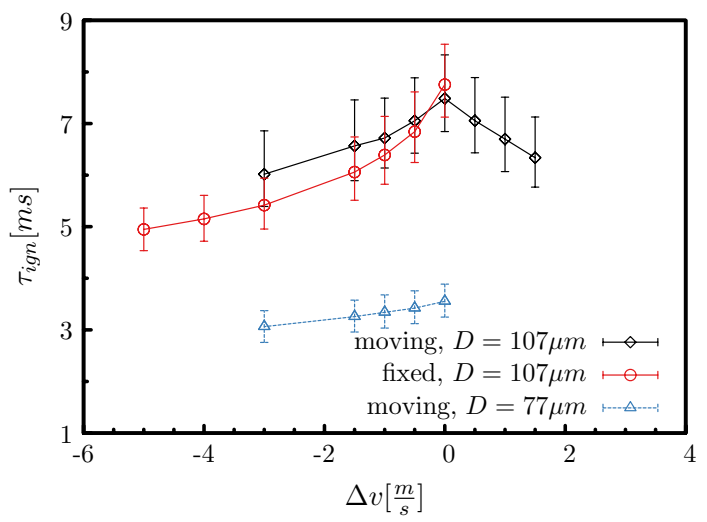


equal to $1.5 \mathrm{~m} / \mathrm{s}$ and the slip velocity is imposed changing the particle velocity. The only exceptions are the cases with large negative slip velocity that are obtained with a gas velocity of $0.5 \mathrm{~m} / \mathrm{s}$ in order to avoid an excessive initial particle velocity. The ignition delay time decreases monotonically if the slip velocity increases for all cases tested. For large slip velocities, the effect appears to saturate. The reason why a saturation occurs is explained in the following, together with the mechanisms responsible for the decreased ignition delay time. The effect is larger for the "fixed particle" cases compared to that in the "moving particle" configuration. This is due to the fact that in the "moving particle" case the slip velocity decreases with time, c.f. Fig. 7, limiting the effect. For the "moving particle" case, the symmetry of the process is also investigated imposing both a positive slip velocity (gas velocity larger than the initial particle velocity) and a negative slip velocity (gas velocity smaller than the initial particle velocity). It is found that the different sign of the slip velocity does not play an important role and an almost symmetric profile is observed. For the "fixed particle" cases, changing the sign of the slip velocity would, of course, have no effect. The small difference observed for a zero slip velocity in the two configuration is due to the fact that in the "moving particle" case the sink of energy in the gas phase due to the particle heating causes an increase of gas density, which is responsible for a small particle deceleration with respect to the fluid and then a small slip velocity. Finally, a different diameter is also considered and shown in Fig. 8; a very similar effect of the slip velocity is observed with a significant decrease of ignition delay time.

There are two processes that might be responsible for the decreased ignition delay time: (i) Due to the sensitivity of ignition to the process of particle heating (Farazi et al. 2019a), the enhanced rate of energy transfer from the gas to the particle due to the slip velocity, i.e., a non-zero particle Reynolds number, might have an effect. This mechanism is due to the dependency of the Nusselt number on the particle Reynolds number, a dependency that is in general included in the model. (ii) Due to the slip velocity and the asymmetric velocity field around the particle location, the particle drifts with respect to the temperature field and is not located exactly in the point where the temperature has its minimum in space. Therefore, the particle is exposed to a gas-phase temperature which is larger compared to the temperature the particle would experience in the case of zero slip.

The relative importance of these two processes cannot be inferred directly from the results obtained employing the whole model. Therefore, in order to assess the effects of the modified particle heating due to a different heat transfer coefficient, a simulation with nonzero slip velocity is performed neglecting the effect of the slip velocity on the heat transfer

Fig. 9 Effect of the inclusion of the dependency of the Nusselt number on the slip velocity on the particle temperature and gas temperature at the particle location: full model (black lines) and model with no effect of slip velocity on the Nusselt number (red lines)

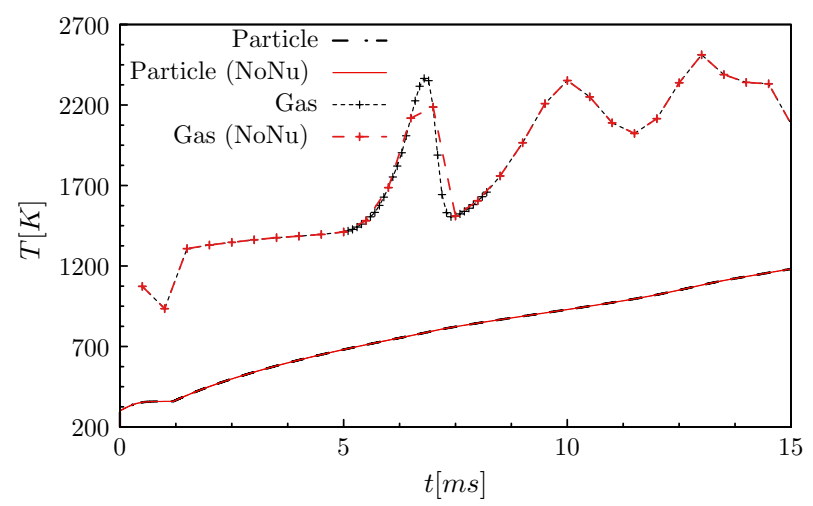


coefficient, i.e., on the Nusselt number. A comparison of this latter case with a simulation at the same conditions but including the effect of the slip velocity on the Nusselt number is shown in Fig. 9. In particular, the comparison of the time evolutions of the particle temperature and that of the gas at the particle position reveals that the inclusion of the effect of the slip velocity on the Nusselt number has a completely negligible impact, with the exception of some minor differences in the gas temperature at the particle location after ignition already happened. As observed in previous studies (Yang et al. 2014; Farazi et al. 2019a, b), the ignition process is characterized by different steps that are related to different phases of devolatilization and causes the variations of the gas-phase temperature at the particle position observed in Fig. 9.

Therefore, the reason for the decreased ignition delay time in the case of a non-zero slip velocity must be the different temperature and species concentration within the flow field surrounding the particle. The temperature profile along a line in the streamwise direction crossing the location of the particle is shown in Fig. 10. The profile is shown at the same physical time in two cases with and without slip velocity. Due to the energy transfer from the gas to the particle, the region around the particle location is characterized by a significantly lower temperature compared to the far field. However, in the case with slip velocity, that in this case is from right to left in the particle frame of reference, an asymmetric temperature field is present around the particle and the particle is exposed to an overall larger value of gas temperature, which promote particle heating and an earlier onset of combustion.

Therefore, it can be concluded that the effect of the slip velocity on ignition delay time is governed by the modification of the temperature field around the particle caused by the relative movement, while the modification to the heat transfer coefficient has a negligible role.

\subsection{Effect of Particle Swelling}

From the experimental images, it is possible to extract the time evolution of the particle diameter. In particular, it is observed that a significant particle swelling occurs over the entire particle trajectories, from the initial particle heating to the ignition and combustion.

In order to assess the effect of particle swelling on the ignition delay time, a series of simulations is performed with the swelling model included in the CPD and described in

Fig. 10 Temperature field around the particle location with (red) and without (black) slip velocity

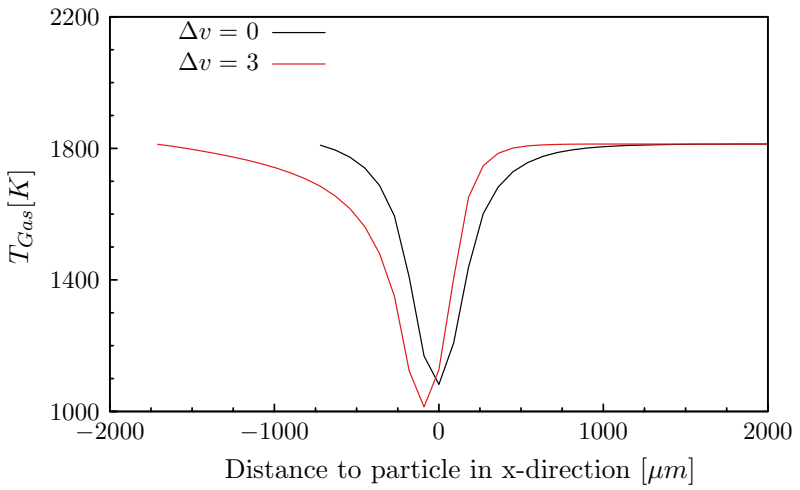


Fig. 11 Time evolution of the particle diameter due to swelling computed using the CPD model (red line) and measured in the experiment for particle ignition with initial diameter of $107 \mu \mathrm{m}$. For the experiment, the line is a fit over all the available measurements of particles with initial diameter in the range $90-125 \mu \mathrm{m}$

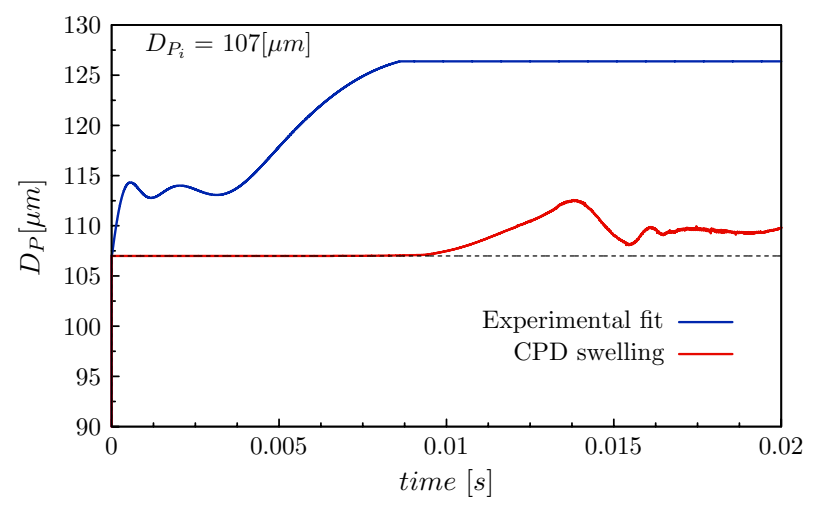

Sect. 2.2. In all the cases presented in this section, the initial particle velocity is prescribed using the average measured value shown in Fig. 7.

The time evolution of the particle diameter for a representative case of a particle with an initial diameter of $107 \mu \mathrm{m}$ is shown in Fig. 11. The simulation result obtained with the CPD swelling model is compared with the measured particle diameter averaged over a set of experiments for particles with similar initial diameter. It is evident that the CPD swelling model is not able to capture the evolution of diameter observed in the experiment. As evident from Eq. 1, the change of particle diameter due to swelling in the CPD model is strongly related to the fraction of labile bridges. Since the fraction of labile bridge does not change significantly before ignition, the diameter of the particle is approximately constant during this phase. This behavior is inconsistent with experiments; from the measurements, it is clearly observed that a remarkable increase of the particle diameter occurs also in the first stages of particle heating, significantly before the ignition. This observation suggests that the chemical swelling included in CPD is not the only effect to be included and additional contribution to swelling related to particle heating or evaporating water should be included. However, the development of a more general model is out of the scope of the present work and will be the focus of a future research effort.

In order to assess the possible effect of an increase of the particle diameter during the heating process, the experimentally measured time evolution of the particle diameter is fitted with an analytical function and prescribed in the simulations. The fitting used has the following form:

$$
\left(\frac{d_{p}}{d_{p_{0}}}\right)=a_{0}+a_{1} e^{-\left(\left(x-b_{1}\right) / c_{1}\right)^{2}}+a_{2} e^{-\left(\left(x-b_{2}\right) / c_{2}\right)^{2}}+a_{3} e^{-\left(\left(x-b_{3}\right) / c_{3}\right)^{2}}+a_{4} e^{-\left(\left(x-b_{4}\right) / c_{4}\right)^{2}}
$$

Since the evolution of the particle diameter observed in the experiment is different for different initial diameter, several fits have been employed for different range of the initial diameter. The fitting corresponding to the measured diameters for particles with an initial diameter in a small range around $107 \mu \mathrm{m}$ is also shown in Fig. 11.

The results for the ignition delay times including the CPD model swelling and with the swelling obtained from experimental fits are shown in Fig. 12. As expected, the swelling modeled in the CPD framework has negligible effects on the ignition delay time because until ignition, the change in diameter is negligible. Prescribing the measured time evolution of particle diameters, which is significant already during the early phase of the particle heating, the ignition delay time computed in the simulations is 
Fig. 12 Ignition delay times measured in the experiment (black diamonds) and computed in the simulations (color symbols) versus the initial particle diameter for several cases employing different swelling models. Each black diamond correspond to the measurement of a single particle ignition. The error bars in the numerical results are defined by the difference in ignition delay time based on different variables and marked by the shaded area in Fig. 4

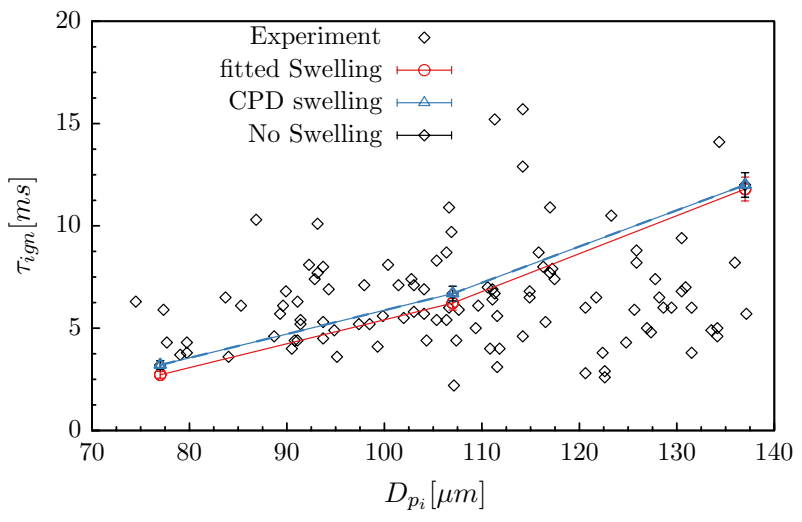

slightly shorter than that obtained neglecting swelling. The effect of swelling on the ignition delay time is more important for small particles, reducing the ignition delay time by about $20 \%$, while the effect becomes almost negligible for large particle sizes. This is due to the higher swelling measured for small particles compared to large particles, which is related to the higher heating rate of small particles.

\section{Conclusions}

Experiments and numerical simulations, based on a Lagrangian point particle framework, were performed for coal particle ignition in a laminar reactor to investigate the effect of initial particle diameter, initial slip velocity, and particle swelling on the ignition delay time. The ignition delay time increases significantly with the increase of the particle diameter, mainly due to the larger time needed for particle heating. It is found that the slip velocity, i.e., the difference between the particle and the gas velocity, has an important effect on the ignition delay time, generally accelerating ignition. It is concluded that this is not related to the different heat transfer rate between the gas and the particle due to the dependency of the Nusselt number on the slip velocity. The effect can be totally ascribed to modifications of the temperature field around the particle, which is caused by the relative velocity. Finally, the effect of particle swelling is analyzed and it is found that the increase of particle diameter due to swelling observed in the experiment is responsible for an acceleration of ignition by about $20 \%$ for small particles while the effect appears to be negligible for large particle sizes.

Acknowledgements The authors kindly acknowledge financial support through Deutsche Forschungsgemeinschaft (DFG) through SFB/TRR 129. The authors gratefully acknowledge the computing time granted through JARA-HPC on the supercomputer JURECA Jülich Supercomputing Centre (2016) and JUQUEEN Jülich Supercomputing Centre (2015) at Forschungszentrum Jülich under project JHPC22 and JHPC48.

Funding Open Access funding enabled and organized by Projekt DEAL.

\section{Compliance with Ethical Standards}

Conflict of interest The authors declare that they have no conflict of interest. 
Open Access This article is licensed under a Creative Commons Attribution 4.0 International License, which permits use, sharing, adaptation, distribution and reproduction in any medium or format, as long as you give appropriate credit to the original author(s) and the source, provide a link to the Creative Commons licence, and indicate if changes were made. The images or other third party material in this article are included in the article's Creative Commons licence, unless indicated otherwise in a credit line to the material. If material is not included in the article's Creative Commons licence and your intended use is not permitted by statutory regulation or exceeds the permitted use, you will need to obtain permission directly from the copyright holder. To view a copy of this licence, visit http://creativecommons.org/licenses/by/4.0/.

\section{References}

Apte, S.V., Mahesh, K., Lundgren, T.: Accounting for finite-size effects in simulations of disperse particle-laden flows. Int. J. Multiph. Flow 34(3), 260-271 (2008). https://doi.org/10.1016/j.ijmultiphaseflo w.2007.10.005

Attili, A., Bisetti, F., Mueller, M.E., Pitsch, H.: Formation, growth, and transport of soot in a three-dimensional turbulent non-premixed jet flame. Combust. Flame 161(7), 1849-1865 (2014). https://doi. org/10.1016/j.combustflame.2014.01.008

Bejarano, P.A., Levendis, Y.A.: Single-coal-particle combustion in $\mathrm{O}_{2} / \mathrm{N}_{2}$ and $\mathrm{O}_{2} / \mathrm{CO}_{2}$ environments. Combust. Flame 153(1), 270-287 (2008)

Bisetti, F., Blanquart, G., Mueller, M.E., Pitsch, H.: On the formation and early evolution of soot in turbulent nonpremixed flames. Combust. Flame 159(1), 317-335 (2012)

Desjardins, O., Blanquart, G., Balarac, G., Pitsch, H.: High order conservative finite difference scheme for variable density low Mach number turbulent flows. J. Comput. Phys. 227(15), 7125-7159 (2008)

Farazi, S., Attili, A., Kang, S., Pitsch, H.: Numerical study of coal particle ignition in air and oxy-atmosphere. Proc. Combust. Inst. 37, 2867-2874 (2019a)

Farazi, S., Hinrichs, J., Davidovic, M., Falkenstein, T., Bode, M., Kang, S., Attili, A., Pitsch, H.: Numerical investigation of coal particle stream ignition in oxy-atmosphere. Fuel 241, 477-487 (2019b)

Farazi, S., Sadr, M., Kang, S., Schiemann, M., Vorobiev, N., Scherer, V., Pitsch, H.: Resolved simulations of single char particle combustion in a laminar flow field. Fuel 201, 15-28 (2017). https://doi. org/10.1016/j.fuel.2016.11.011

Goshayeshi, B., Sutherland, J.C.: A comparison of various models in predicting ignition delay in single-particle coal combustion. Combust. Flame (2014). https://doi.org/10.1016/j.combustflame.2014.01.010

Grant, D.M., Pugmire, R.J., Fletcher, T.H., Kerstein, A.R.: Chemical model of coal devolatilization using percolation lattice statistics. Energy Fuels 3(2), 175-186 (1989). https://doi.org/10.1021/ef00014a011

Jimenez, S., Gonzalo-Tirado, C.: Properties and relevance of the volatile flame of an isolated coal particle in conventional and oxy-fuel combustion conditions. Combust. Flame 176, 94-103 (2017)

Jupudi, R.S., Zamansky, V., Fletcher, T.H.: Prediction of light gas composition in coal devolatilization. Energy Fuels 23(6), 3063-3067 (2009). https://doi.org/10.1021/ef9001346

Jülich Supercomputing Centre. JURECA: General-purpose supercomputer at Jülich Supercomputing Centre. J. Large-Scale Res. Facil. 2016. https://doi.org/10.17815/jlsrf-2-121

Jülich Supercomputing Centre. JUQUEEN: IBM Blue Gene/Q Supercomputer System at the Jülich Supercomputing Centre. J. Large-Scale Res. Facil. 1(A1), 2015. https://doi.org/10.17815/jlsrf-1-18

Khatami, R., Stivers, C., Levendis, Y.A.: Ignition characteristics of single coal particles from three different ranks in $\mathrm{O}_{2} / \mathrm{N}_{2}$ and $\mathrm{O}_{2} / \mathrm{CO}_{2}$ atmospheres. Combust. Flame 159(12), 3554-3568 (2012)

Köser, J., Becker, L.G., Goßmann, A.-K., Böhm, B., Dreizler, A.: Investigation of ignition and volatile combustion of single coal particles within oxygen-enriched atmospheres using high-speed oh-plif. Proc. Combust. Inst. 36(2), 2103-2111 (2017). https://doi.org/10.1016/j.proci.2016.07.083

Köser, J., Li, T., Vorobiev, N., Dreizler, A., Schiemann, M., Böhm, B.: Multi-parameter diagnostics for high-resolution in-situ measurements of single coal particle combustion. Proc. Combust. Inst. 37(3), 2893-2900 (2019). https://doi.org/10.1016/j.proci.2018.05.116

Li, T., Schiemann, M., Köser, J., Dreizler, A., Böhm, B.: Experimental investigations of single particle and particle group combustion in a laminar flow reactor using simultaneous volumetric oh-lif imaging and diffuse backlight-illumination. Renew. Sustain. Energy Rev. (under review) (2020)

Liu, Y., Geier, M., Molina, A., Shaddix, C.R.: Pulverized coal stream ignition delay under conventional and oxy-fuel combustion conditions. Int. J. Greenh. Gas Control 5, S36-S46 (2011). https://doi. org/10.1016/j.ijggc.2011.05.028 
Maffei, T., Khatami, R., Pierucci, S., Faravelli, T., Ranzi, E., Levendis, Y.A.: Experimental and modeling study of single coal particle combustion in $\mathrm{O}_{2} / \mathrm{N}_{2}$ and Oxy-fuel $\left(\mathrm{O}_{2} / \mathrm{CO}_{2}\right)$ atmospheres. Combust. Flame 160(11), 2559-2572 (2013)

Miller, R.S., Bellan, J.: Direct numerical simulation of a confined three-dimensional gas mixing layer with one evaporating hydrocarbon-droplet-laden stream. J. Fluid Mech. 384(3), 293-338 (1999). https://doi. org/10.1017/S0022112098004042

Molina, A., Shaddix, C.R.: Ignition and devolatilization of pulverized bituminous coal particles during oxygen/carbon dioxide coal combustion. Proc. Combust. Inst. 31(2), 1905-1912 (2007)

Riaza, J., Khatami, R., Levendis, Y.A., Álvarez, L., Gil, M.V., Pevida, C., Rubiera, F., Pis, J.J.: Single particle ignition and combustion of anthracite, semi-anthracite and bituminous coals in air and simulated oxy-fuel conditions. Combust. Flame 161(4), 1096-1108 (2014)

Shaddix, C.R., Molina, A.: Particle imaging of ignition and devolatilization of pulverized coal during oxyfuel combustion. Proc. Combust. Inst. 32(2), 2091-2098 (2009)

Shurtz, R.C., Hogge, J.W., Fowers, K.C., Sorensen, G.S., Fletcher, T.H.: Coal swelling model for pressurized high particle heating rate pyrolysis applications. Energy Fuels 26(6), 3612-3627 (2012)

Shurtz, R.C., Kolste, K.K., Fletcher, T.H.: Coal swelling model for high heating rate pyrolysis applications. Energy Fuels 25(5), 2163-2173 (2011)

Smith, G.P. et al.: GRI 3.0. http://www.gaseq.co.uk/. Accessed May 2017

Tufano, G.L., Stein, O.T., Kronenburg, A., Frassoldati, A., Faravelli, T., Deng, L., Kempf, A.M., Vascellari, M., Hasse, C.: Resolved flow simulation of pulverized coal particle devolatilization and ignition in airand $\mathrm{O}_{2} / \mathrm{CO}_{2}$-atmospheres. Fuel 186, 285-292 (2016)

Vorobiev, N., Geier, M., Schiemann, M., Scherer, V.: Experimentation for char combustion kinetics measurements: bias from char preparation. Fuel Process. Technol. 151, 155-165 (2016). https://doi. org/10.1016/j.fuproc.2016.05.005

Yang, H., Li, S., Fletcher, T.H., Dong, M., Zhou, W.: Simulation of the evolution of pressure in a lignite particle during pyrolysis. Energy Fuels 28, 3511-3518 (2014)

\section{Affiliations}

\section{Antonio Attili ${ }^{1}$ D $\cdot$ Pooria Farmand ${ }^{1} \cdot$ Christoph Schumann $^{1} \cdot$ Sima Farazi $^{1}$. Benjamin Böhm² $\cdot$ Tao Li $^{2}$. Christopher Geschwindner² · Jan Köser² . Andreas Dreizler ${ }^{2} \cdot$ Heinz Pitsch ${ }^{1}$}

\section{Antonio Attili}

a.attili@itv.rwth-aachen.de

1 Institute for Combustion Technology, RWTH Aachen University, 52056 Aachen, Germany

2 Institute for Reactive Flows and Diagnostics, Technische Universität Darmstadt, Darmstadt, Germany 